\title{
Les fumeurs d'opium à Canton dans les années 1930
}

\section{Xavier Paulès*}

Dans les dernières années de l'Empire, l'opium est d'abord une question de politique extérieure pour la Chine qui s'efforce d'obtenir par la négociation la fin des importations en provenance d'Inde. Mais sur le plan de la politique intérieure, son éradication constitue aussi un des objectifs de la politique de réforme menée à partir de 1901-1902. Le plan de suppression progressive et générale de la drogue mis en place par les Qing à partir de 1906 peut s'appuyer sur le succès diplomatique que représente l'engagement pris dès l'année suivante par la Grande-Bretagne de réduire année par année ses exportations d'opium. Le plan se traduit par un recul très significatif des surfaces plantées en pavot et un fléchissement notable de la demande.

Toutefois, la fin de l'Empire et surtout l'effondrement du pouvoir central marquent un coup d'arrêt. La nouvelle donne politique provoque un

* Xavier Paulès est doctorant à l'Institut d'Asie Orientale (Lyon). Sa thèse est intitulée L'opium à Canton pendant la période républicaine (1912-1937). Essais de mainmise politique et pratiques sociales. L'auteur tient à remercier ici vivement les personnes qui lui ont apporté des critiques et suggestions pour l'amélioration de cet article : C. Henriot, H. Delahaye et $\mathrm{C}$. Chevaleyre, ainsi que la fondation Chiang Ching-kuo pour la bourse dont il bénéficie dans sa dernière année de thèse. 
regain de la production et de la consommation. Très vite, sous la République, l'opium reprend une importance politique essentielle. Mais il n'est plus alors un enjeu diplomatique ou une partie d'un programme de renouveau national: il joue désormais le rôle d'un catalyseur du processus d'émiettement politique du pays. Les diverses taxes sur le commerce et les fumeries d'opium deviennent en effet, à partir du milieu des années 1910, une source capitale de rentrées fiscales pour les différents seigneurs de la guerre qui s'émancipent rapidement du pouvoir central.

Dans ce contexte, la mainmise sur les revenus de l'opium constitue une nécessité pour quiconque espère réunifier le pays. Le Guomindang, dont c'est là précisément l'ambition, entreprend progressivement, à partir de 1924, de priver ses adversaires de ces revenus. Dans le même temps, il s'efforce, avec un certain succès, d'en tirer lui-même les plus grands profits possibles afin de financer son programme.

L'importance indiscutable que l'opium revêt donc sur le plan politique dans la Chine républicaine a, très logiquement, incité les historiens à lier l'étude de cette drogue à la problématique de l'unification nationale menée par le Guomindang. Sa volonté de mainmise sur les circuits de l'opium, qui se traduit par l'élaboration de différents systèmes de contrôle de plus en plus efficaces a, par conséquent, déjà donné lieu à de nombreuses études ${ }^{1}$. On peut même affirmer que d'une façon plus générale, les historiens ont jusqu'ici privilégié l'étude de l'offre, c'est à dire celle de la production et de la distribution par rapport à celle de la demande.

1 Edward R. Slack, Opium, State and society, China's narco-economy and the Guomindang, 1924-1937, Honolulu : University of Hawaï Press, 2001 ; Alan Baumler, Playing with fire: the Nationalist government and opium in China, 1927-1941, thèse de doctorat, Université de l'Illinois, 1997 ; Yongming Zhou, Anti-drug crusades in twentieth-century China: nationalism, history and state building, Lanham : Rowman \& Littlefield, 1999, ou encore certains chapitres de l'ouvrage collectif remarquable de Timothy Brook et Bob Tadashi Wakabayashi (éd.), Opium regimes, China, Britain and Japan, 1839-1952, Berkeley : University of California Press, 2000. 
On constate cependant, depuis quelques années, un certain intérêt pour les questions relatives à la consommation d'opium ${ }^{2}$. L'étude des populations de fumeurs, qui devrait logiquement accompagner cette nouvelle tendance, est néanmoins restée presque complètement négligée. On pourrait tenter d'expliquer cette carence par le défaut de sources d'information, mais ce problème ne constitue pas en réalité un obstacle insurmontable pour l'étude des fumeurs. Si le peu d'études qui ont été menées sur la question ont souvent été peu satisfaisantes, c'est parce qu'elles prétendaient concerner l'ensemble du pays, alors que c'est précisément à cette échelle que les sources fiables sont les moins nombreuses.

Ainsi, certains chercheurs tentent d'estimer le nombre total des fumeurs en Chine au début $\mathrm{du} \mathrm{XX}^{\mathrm{e}}$ siècle. Mais leur méthode demeure la même que celles des diplomates occidentaux de l'époque. Elle consiste à diviser la quantité écoulée une année donnée sur le marché par la consommation annuelle d'un fumeur considéré comme moyen ${ }^{3}$. Or, il est évident qu'un tel calcul pose un problème de rigueur, ne serait-ce que parce que la définition des deux valeurs de ce quotient ne va pas du tout de soi. L'estimation d'une consommation annuelle moyenne par fumeur est une véritable gageure et elle devrait inciter à la plus grande circonspection quant à la fiabilité des résultats obtenus.

D'autres historiens cherchent, toujours au niveau national, à décrire de façon qualitative (âge, sexe, métier...) qui sont les fumeurs. Mais pour

2 Keith Mac Mahon, "Opium and sexuality in late Qing fiction", Nannü, vol. 2, $\mathrm{n}^{\circ} 1$ (2000), p. 129-179 ; Lars P. Laamann, "A history of narcotic consumption in modern China", Twentieth-Century China, vol. 28, n०1 (novembre 2002), p. 1-20; Xun Zhou, "Pain and pleasure: Opium as medicine in late imperial China", Twentieth-Century China, vol. 28, $\mathrm{n}^{\circ} 1$ (novembre 2002), p. 21-36.

${ }^{3}$ Le Report of the International anti-opium commission, Shanghai : North China Daily News, 1909, p. 67-68, par exemple, obtient par cette méthode le chiffre de 13,5 millions de fumeurs pour 1908. Pour les études récentes, voir : R. K. Newman, "Opium smoking in late imperial China: A reconsideration", Modern Asian Studies, vol. 29.4 (octobre 1995), p. 769, et aussi : Wang Hongbin 王厷斌, Jindu shinian 禁毒史鍳 (Histoire de la prohibition des drogues), Changsha: Yuelu shushe, 1997, p. 258. 


\section{Xavier Paulès}

ce faire, ils ne s'appuient que sur les chiffres de l'Association Nationale Anti-Opium (ANAO) ${ }^{4}$, sans les soumettre à une critique suffisante ${ }^{5}$. Les chiffres fournis par cette association souffrent de l'opacité qui entoure leur collecte et fourmillent d'erreurs ${ }^{6}$. Au-delà des coquilles et fautes de calcul, leur irréalisme est patent en certains endroits. Lorsque la chose est précisée, on apprend qu'ils sont constitués par simple agrégation d'estimations de correspondants locaux plus ou moins éclairés à partir desquelles sont extrapolés des résultats généraux ${ }^{7}$. Cela n'a du reste rien d'étonnant: l'ANAO, en dépit de son implantation nationale, ne dispose pas de l'appareil bureaucratique nécessaire pour fournir une description correcte des caractéristiques de la population des fumeurs d'opium du pays. La raison pour laquelle ces données si douteuses ont été néanmoins reprises par des historiens est qu'elles constituent les seules estimations existantes à

${ }^{4}$ En chinois: Zhonghua guomin juduhui 中華國民拒毒會 (Association nationale chinoise anti-opium). Cette association, fondée le 5 août 1924, mobilise en premier lieu les élites de Shanghai. Les Chinois convertis au christianisme y sont particulièrement actifs. L'ANAO se dote rapidement d'un réseau de correspondants et de branches dans toute la Chine et se montre capable de mobiliser des effectifs considérables lors des manifestations qu'elle organise dans le pays.

5 Edward R. Slack, op. cit., p. 42. Su Zhiliang 蘇智良, Zhongguo dupin shi 中國毒品史 (Histoire des stupéfiants en Chine), Shanghai : Shanghai renmin chubanshe, 1997, reproduit p. 333, sans la moindre observation, les chiffres d'une enquête de l'ANAO qui donnent, pour la proportion de fumeurs ayant entre 30 et 50 ans, $61,7 \%$ en 1928 et $36 \%$ l'année suivante.

${ }^{6}$ Parmi d'innombrables exemples, dans le numéro $n^{\circ} 47$ (mars 1931) de la revue $J u d u$ yuekan, 拒起月刊 (Mensuel pour la suppression des drogues) p. 38, lorsque l'on additionne les pourcentages, pour le pays entier, de fumeurs ayant moins de 30 ans, de 30 à 50 ans, et plus de 50 ans, on obtient $92,5 \%$.

${ }^{7}$ Par exemple Zhonghua guomin juduhui 中華國民拒毒會, Zhongguo yanhuo nian-

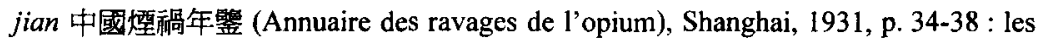
chiffres donnés comme valables au niveau national ont été collectés dans des zones correspondant à une population de seulement 12 millions d'habitants. 
l'échelle du pays ${ }^{8}$. Les statistiques nationales, appuyées sur un pouvoir de coercition, font donc défaut sur cette question, à l'exception de quelques maigres données sur les délits liés à l'opium ${ }^{9}$. De même, le recensement général des fumeurs décidé en 1934 dans le cadre de l'adoption par le Guomindang du plan de six ans pour la suppression progressive de l'opium ne livre malheureusement pas, au niveau national, d'informations autres que le nombre de fumeurs recensés, soit plus de 4,1 millions en 1936-37, et leur répartition par provinces ${ }^{10}$.

Il n'existe donc pas, sous la République, de données nationales fiables permettant d'éclairer des questions aussi élémentaires que la structure par sexe et par âge de ce groupe ou la proportion en son sein des différentes catégories socio-professionnelles. Pourtant, cela ne signifie pas que l'on doive renoncer à toute recherche : la question doit simplement être traitée à un niveau plus modeste, et le seul qui semble pertinent dans l'état actuel des sources disponibles est celui d'une grande ville comme Canton.

Canton est tout d'abord dotée, dans les années 1930, et comme toutes les grandes métropoles chinoises de la période, d'une presse abondante. Un journal, le Yuehuabao 越華報 (Le méridional), présente la particularité de s'intéresser tout spécialement aux faits divers liés à l'opium. Il s'agit d'une gazette populaire créée en 1927 dont un grand nombre de numéros de la période 1930-38 nous sont parvenus. On peut s'appuyer également sur la presse en langue chinoise de Hong Kong, dont les quotidiens possèdent

\footnotetext{
${ }^{8}$ Zhu Qingbao 朱慶葆, Jiang Qiuming 蔣秋明, Zhang Shijie 張士杰, Yapian yu jindai zhongguo 鴉片與近代中國 (L'opium et la Chine moderne), Nanjing : Jiangsu jiaoyu chubanshe, 1995, p. 197, conclut plus sagement à l'absence de chiffres fiables concernant le nombre total des fumeurs d'opium sous la République.

${ }^{9}$ Guomin zhengfu zhujichu tongjiju 國民政府主計處統計局, Zhonghua minguo tongji tiyao 中華民國統計提要 (Résumé statistique de la République de Chine), Nanjing, 1935, p. 164-71.

${ }^{10}$ Archives nationales $\mathrm{n}^{\circ} 2$ (Nanjing), dossier 41/78: Ershiliu niandu jinyan nianbao, 二十六年度禁煙年報 (Rapport annuel sur la prohibition de l'opium, année 1937), p. 53.
} 


\section{Xavier Paulès}

l'avantage d'une plus grande liberté de ton vis à vis des autorités cantonaises. Ils mènent de surcroît diverses enquêtes sur la question de l'opium à Canton dans les années $1930^{11}$.

Autre atout de Canton, un comité de suppression de l'opium de la ville, le Guangzhoushi jinyan weiyuanhui 廣州市禁煙委員會, y est créé par les autorités du Guomindang le 28 septembre $1936{ }^{12}$ suite à leur retour au Guangdong ${ }^{13}$. Cet organisme se révèle assez actif et fournit quelques mois plus tard les chiffres d'un recensement des fumeurs effectué en 19361937.

Le comité fonde aussi un institut de désintoxication (Guangzhoushi jieyan yiyuan 廣州市戒煙醫院), inauguré en mars 1937, rue Yide (yide lu 一德路) à Canton, et qui fonctionne jusqu'à l'arrivée des Japonais. Un autre institut du même genre (Guangdong jinyan liuyisuo 廣東禁煙留醫所) est créé par le gouvernement collaborationniste en janvier 1941. Tous les deux ont publié des rapports ${ }^{14}$ qui fournissent des détails précis, parfois même

${ }^{11}$ Deux journaux se distinguent pour l'abondance des informations qu'ils fournissent sur la situation de l'opium à Canton au milieu des années 1930 : le Xianggang gongshang ribao 香港工商日報 (Quotidien de l'industrie et du commerce de Hong Kong) et le Xunhuan ribao 循環日報 (La circulation).

12 Guangzhoushi jinyan weiyuanhui 廣州市禁煙委員會, Guangzhoushi jinyan weiyuanhui gongzuo jiyao 廣州市禁煙委員會工作紀要 (Résumé des activités du comité de suppression de l'opium de la ville de Canton), Canton, 1937, ci-après GJWGJ), p. 87.

${ }^{13}$ Elles délogent en juillet 1936 Chen Jitang 陳濟棠 qui dirigeait la province en toute indépendance vis-à-vis du gouvernement de Nankin depuis 1931.

${ }^{14}$ Guangzhoushi jinyan weiyuanhui 爰州市禁煙委員會, Guangzhoushi jieyan yiyuan nianbao 廣州市戒煙醫院年報 (Rapport annuel sur la clinique de désintoxication de Canton), Canton, 1937 (ci-après $G J Y N$ ) et Guangdong jinyan liuyisuo 廣東禁煙留醫所, Guangdong jinyan liuyisuo chengli yi zhounian jinian tekan, 廣東禁煙留醫所成立一週年紀念特刊 (Édition spéciale pour commémorer le premier anniversaire de la fondation de l'institut de désintoxication du Guangdong), Canton, 1942 (avril), ci-après GJL. Dans la suite de cet article, afin de prévenir toute 
sur une base individuelle, sur les patients qui y ont été soignés. Ils ont hébergé chacun au bas mot mille personnes durant la période d'activité dont rendent compte les rapports, c'est-à-dire, pour le premier, de mars à juin 1936 et pour le second, de janvier 1941 à mars 1942. Ces trois principales sources, confrontées et étayées par d'autres plus secondaires, permettent de définir pour la première fois et avec une certaine rigueur qui étaient les fumeurs d'opium de Canton.

Nous allons voir que le trait caractéristique de cette population est sa concentration dans certaines catégories de la population, et qu'il existe bien un fumeur type : il est de sexe masculin, travailleur manuel et d'âge mûr. Le tireur de pousse représentant une sorte d'archétype de cette catégorie de la population adonnée à l'opium. Nous le prendrons comme exemple pour présenter rapidement dans quelles conditions matérielles il consomme quotidiennement sa drogue.

Précisons que cet article exclut l'étude des mentalités des fumeurs d'opium de Canton, non moins nécessaire pour espérer comprendre qui ils étaient, mais dont le traitement n'est pas possible dans les limites de cette publication.

Enfin, il n'est pas inutile de signaler qu' à Canton, avant la chute de Chen Jitang en 1936, la consommation d'opium n'est pas soumise à la détention d'un permis spécial, pourvu qu'elle concerne le yangao 煙膏 officiel et s'effectue dans les fumeries légales ${ }^{15}$. A partir de 1936, cependant, il devient indispensable de se faire recenser. Le fumeur enregistré reçoit un permis individuel qui spécifie la quantité d'opium qu'il peut fumer chaque jour.

confusion possible, nous nommerons ces deux établissements en faisant référence à leur année de fondation, c'est-à-dire 1937 et 1941.

${ }^{15}$ Le cas des fumeuses est particulier : la fréquentation des fumeries leur est interdite à partir de 1932. 


\section{Xavier Paulès}

\section{Les contours problématiques d'une population}

\section{Qu'est ce qu'un fumeur d'opium ?}

Derrière l'évidence du terme "fumeur d'opium " se cache le problème, vital pour notre étude, de sa définition rigoureuse. Les sources, lorsqu'elles s'essaient à estimer le nombre de fumeurs, n'envisagent pas la question du minimum à partir duquel on peut être considéré comme un fumeur et non plus un simple profane. À partir des données fournies par l'institut de 1937 pour les patients accueillis entre mars et juin 1937, on constate pourtant qu'une grande partie des fumeurs ne consomment chaque jour qu'une quantité très modeste de drogue (la majorité fumait moins d'un qian 錢, soit $3,8 \mathrm{~g}$ par jour, quantité qui correspond à six ou sept pipes pour une durée de consommation d'une petite demi-heure $)^{16}$. La question du continuum avec la population des non fumeurs est donc posée : où classer l'individu qui ne fume que quelques pipes de temps en temps pour accompagner ses amis?

Les consommateurs d'opium établissent bien, eux, dans l'espace étriqué des rares sources où peut s'exposer leur vision des choses, une distinction assez nette entre les fumeurs d'opium en général et les opiomanes proprement dits, qui n'en constituent qu'un sous groupe. Ainsi, un certain nombre (de 7 à $10 \%$ ) des individus qui rentrent à l'institut de 1937 refusent tout simplement de se dire malades, probablement parce qu'ils considèrent qu'ils ne sont pas dépendants ou que leur consommation ne peut nuire à leur santé ${ }^{17}$. On voit par là que pour eux, au sein du groupe des fumeurs, la démarcation opiomane / fumeur occasionnel (on aimerait pouvoir dire « fumeur du dimanche ») existe.

En français, les deux termes fumeur occasionnel et "opiomane" rendent compte de la possibilité d'être ou non un consommateur dépendant. Par contre, les appellations qui prolifêrent dans les publications de

\footnotetext{
${ }^{16} G J Y N$, partie statistique, p. 8.

${ }^{17} G J Y N$, partie statistique, p. 5 et 7-10.
} 
l'époque et la presse cantonaise, qui considèrent toutes l'opium comme un fléau, ont tendance à pratiquer l'amalgame. Les termes les plus usités y sont: yangui 煙鬼 (les diables fumeurs d'opium), daoyou 道友 (les initiés), zhuangshi 壯士 (les braves), furong xianzi 芙蓉仙子 (les esprits du pavot), yin junzi 疲君子 (les Messieurs dépendants), xian 仙 (les immortels), heiji zhongren 黑籍中人 (les damnés). On constate en tout cas dans cette liste l'absence d'un mot spécifique pour définir le fumeur occasionnel. Un article du Yuehuabao du 21 mars 1931 est intéressant à cet égard : la présence de jeunes femmes chargées du service ayant été frappée d'interdiction quelques jours avant, le journal relate la disparition des fumeries d'une certaine catégorie de clients dont l'intérêt se portait plus sur elles que sur la drogue. Pour désigner ces personnes, le journaliste est contraint par l'absence de terme adéquat d'utiliser une périphrase amusante : feizhengshi daoyou 非正式道友 ( (es fumeurs non officiels»). Plus généralement, les articles des faits divers du Yuehuabao ne précisent le degré de dépendance des fumeurs, lorsqu'ils l'évoquent, que pour le décrire comme très élevé. De même, beaucoup des termes désignant les fumeurs filent la métaphore qui assimile les fumeurs à des êtres divins. Et ces mêmes articles se plaisent à opposer ironiquement le monde des fumeurs à celui des gens ordinaires sur le mode d'une opposition du profane ( $f a n$ 凡) et du sacré (xian 仙) ${ }^{18}$.

La conclusion qu'il faut tirer de toutes ces remarques est que les sources tendent à considérer sans la moindre nuance tous les fumeurs, si modeste et irrégulière leur consommation soit-elle, et à estimer qu'ils sont tous sous la dépendance de la drogue pour mieux les opposer au reste de la population. Cette logique d'agrégation est clairement à l'œuvre dans le fonctionnement même de l'institut fondé en 1937. C'est du reste, beaucoup plus largement, celle des autorités d'alors ${ }^{19}$.

18 Parmi de nombreux exemples, voir les articles du Yuehuabao 5/8/31,21/12/31, $2 / 2 / 32,4 / 2 / 32$.

${ }^{19}$ Xavier Paulès, " Guérir, éduquer, délimiter : la clinique pour opiomanes de Canton en $1937 »$, Les cahiers d'histoire, $\mathrm{n}^{\circ}$ 3-4 (2002). 


\section{Xavier Paulès}

Étant prisonniers, pour une large part, des chiffres fournis par elles, notre définition d'un fumeur sera donc également cette définition " large » et officielle. Par opposition, nous n'utiliserons le terme d'opiomane que dans son sens le plus strict, c'est-à-dire une personne identifiée comme étant sous la dépendance de l'opium.

\section{Quelle part de la population totale?}

Rares sont les contemporains concernés qui se sont risqués à évaluer le nombre de fumeurs dans la population cantonaise de cette période. En décembre 1936, le Xianggang gongshang ribao 香港工商日報 ${ }^{20}$, citant un officiel du Bureau de gestion des fumeries de Canton (Guangzhoushi shouxisuo guanlichu 廣州市售吸所管理處), avance le nombre de 80000 . Son confrère le Xunhuan ribao 循環日報 ${ }^{21}$, relayant très vraisemblablement des informations émanant du même organisme, le place lui dans une fourchette allant de 70000 à 80000 . L'année suivante, en juin, le directeur de l'institut de désintoxication l'estime à $40000^{22}$ et la revue Judu yuekan 拒毒月刊 avance le chiffre de $30000^{23}$.

Se détachant de ces estimations intuitives, le recensement des fumeurs de 1936-37 mené par le comité de suppression de l'opium de la ville de Canton s'appuie, lui, sur un pouvoir de contrainte réel. Sa finalité et ses règles sont définies avec précision. Il constitue une source essentielle, ce qui ne signifie pas néanmoins qu'elle soit exempte de tout reproche comme nous le verrons.

En mai 1937, après une succession de délais de grâce successifs et une campagne vigoureuse de contrôles et d'inscriptions forcées, on obtient le chiffre de 21721 inscrits $^{24}$. Cette valeur constitue pourtant vraiment un

\footnotetext{
${ }^{20}$ Xianggang gongshang ribao 10/12/36.

${ }^{21}$ Xunhuan ribao 29/12/36.

${ }^{22} G J Y N$, p. 2.

${ }^{23}$ Judu yuekan, $\mathrm{n}^{\circ} 113$ (juin 1937), p. 16.

${ }^{24}$ GJWGJ, p. 16.
} 
minimum, non seulement en regard des estimations évoquées plus haut, mais aussi dans la mesure où le recensement donne des chiffres incontestablement trop bas pour certains arrondissements périphériques comme Dongshan 東山 ou Huadi 花地 ${ }^{25}$. Plus généralement, de nombreux fumeurs sont évidemment tentés de ne pas se faire recenser, espérant s'approvisionner à moindre coût au marché noir ${ }^{26}$. Au début de l'année 1937, un certain nombre parmi eux (997 entre mars et juin 1937) sont interpellés et inscrits de force ${ }^{27}$. Beaucoup d'autres ont eu plus de chance et sont restés dans l'illégalité sans être inquiétés. Cette tendance générale à se soustraire au recensement s'ajoute donc aux insuffisances criantes qui caractérisent plus spécifiquement le recensement dans certains arrondissements.

Au total, il parait vraisemblable que le nombre réel de fumeurs soit plus proche, à la mi-1937, des estimations de $30 / 40000$ que de $80000^{28}$ : constatant la détermination dont font preuve les nouvelles autorités ${ }^{29}$, certains fumeurs occasionnels renoncent en effet à la drogue dans la période 1936-1937.

Un effectif de 21721 ne représenterait, si on évalue la population cantonaise de la fin des années 1930 à 1,189 millions d'habitants, que $1,83 \%$ de la population totale de la ville. Nombre peu impressionnant, il s'agit là d'une valeur probablement plus proche du pourcentage des vrais opiomanes que de celui des fumeurs. Si l'on retient 40000 comme l'ordre de grandeur le plus crédible du nombre de fumeurs cantonais, ce sont alors $3,36 \%$ de la population qui fument l'opium. Ce pourcentage représente

\footnotetext{
${ }^{25}$ Par exemple, le faubourg de Huadi comptabilise une unique personne recensée, pour une population de plus de 15000 âmes : GJWGJ, annexes, p. 3.

${ }^{26} \mathrm{Ce}$ dernier est fort prospère, car l'opium licite que les fumeurs enregistrés sont autorisés à consommer est lourdement taxé.

${ }^{27}$ G.JWGJ, p. 18.

${ }^{28}$ On constate du reste que les estimations hautes datent de la fin de l'année 1936 alors que les estimations tournant autour de 40000 sont postérieures d'une demi-année.

${ }^{29}$ Yuehuabao 20/11/36.
} 


\section{Xavier Paulès}

somme toute une proportion modeste qui doit nous inciter à relativiser l'impact de la consommation d'opium parmi l'ensemble de la population.

\section{Qui sont les fumeurs?}

\section{L'âge}

Commençons l'étude des caractéristiques des fumeurs de Canton par leur âge. On dispose pour cela de deux séries de données essentielles : l'âge de chacun des 171 fumeurs traités en décembre 1941 à la clinique fondée la même année, et la répartition par tranches d'âges de 1128 patients de l'institut de 1937. On constate que la population des deux instituts se recrute en grande majorité parmi les adultes de $25-45$ ans, qui représentent $73,4 \%$ des patients de la clinique de 1937 et $80,7 \%$ de celle de $1941^{30}$. Les graphiques A et B qui mettent ces résultats en rapport avec la structure par âge de la population masculine ${ }^{31}$ de Canton selon le recensement de 1928 montrent l'évidente concentration des fumeurs dans cette tranche d'âge. Les jeunes adultes sont notoirement peu représentés et les personnes âgées ne sont pas particulièrement réceptives à la drogue non plus ${ }^{32}$.

Le recensement des fumeurs en 1937 comporte lui aussi une répartition par tranches d'âges qui corrobore tout à fait ces premiers constats : les

\footnotetext{
${ }^{30} G J Y N$, partie statistique, p. 3 ; GJL, p. 27-40.

${ }^{31}$ La comparaison avec la population cantonaise masculine est justifiée par le fait que la population des deux instituts est quasi-exclusivement ( $99 \%$ et $97,1 \%$ ) composée d'hommes entre 20 et 60 ans.

${ }^{32}$ Les personnes les plus âgées ont, semble-t-il, tendance à fuir le traitement comme le montrent les données sur les 171 patients de la clinique 1941 : l'âge moyen des gens qui y sont conduits de force est de 42 ans quand celui de l'ensemble des patients n'est que de 38,6 ans : $G J L$, p. $27-40$.
} 
$30-45$ ans y représentent à eux seuls $63,3 \%$ des inscrits ${ }^{33}$. De même, sur les 111 personnes interpellées pour des délits liés à la consommation d'opium relatés dans le Yuehuabao entre 1930 et 1936 et pour lesquelles l'âge est spécifié, on compte $64,9 \%$ de $25-45$ ans. Canton n'est de ce point de vue très probablement pas un cas particulier, du moins en Chine du Sud. Certains témoins interrogés par la commission d'enquête pour la question de l'opium en Extrême-Orient en 1929-30 dans les deux autres grands ports du delta de la Rivière des perles, Hong Kong et Macao, décrivent la même désaffection parmi les jeunes ${ }^{34}$.

La nette convergence de ces différentes sources nous paraît garantir la solidité d'un constat qui contredit tout à fait certains discours récurrents de l'époque. Nombre d'articles de presse s'indignent en effet de voir les jeunes gens très nombreux, selon eux, céder aux charmes mortels de l'opium $^{35}$. C'est bel et bien le contraire qui semble vrai.

\section{Commencer à fumer}

Il faut préciser d'emblée que les données fiables manquent malheureusement concernant l'âge auquel on commence à fumer, une question pourtant importante. Par contre, il est possible de prendre position dans un débat intéressant qui concerne la raison pour laquelle on commence à fumer. Les contemporains aussi bien que les historiens actuels ont dégagé deux options possibles ${ }^{36}$ : le pur agrément ou l'usage thérapeutique. Un bon

${ }^{33}$ GJWGJ, partie statistique p. 5-6. Nous sommes malheureusement incapables d'en tirer les chiffres de la même tranche 25-45 que pour les cliniques du fait de l'existence d'une malencontreuse catégorie « moins de 30 ans ».

${ }^{34}$ Archives de la SDN, dossiers S 196 et S 197, interviews de William Edward Leonard Shenton (avocat) du 28 janvier 1930 et du docteur Jose Gaetano Soares, du 23 janvier 1930.

${ }^{35}$ Xianggang gongshang ribao 20/6/35, Yuehuabao 15/1/32, 4/2/32, 27/7/34.

${ }^{36} \mathrm{La}$ question des raisons qui motivent la consommation de drogue est évidemment infiniment plus complexe que ne le laisse croire cette présentation dichotomique. Pour 


\section{Xavier Paulès}

exemple de consommation d'agrément est fourni par ce marchand cantonais, dont la femme vieillissante refuse qu'il prenne une concubine, et qui commence à fumer pour fuir les tracas de son foyer ${ }^{37}$. Par contre, ce coolie de Hong Kong qui commence à fumer l'opium pour soulager des douleurs à la poitrine utilise, lui, l'opium comme un médicament ${ }^{38}$.

S'il est assez facile de comprendre pourquoi des Cantonais commencent à fumer pour le plaisir, il faut exposer par contre pourquoi certains recourent à l'opium pour se guérir. Les effets analgésiques fameux de l'opium procurent évidement un soulagement de la douleur qui peut sûrement passer dans l'esprit de certaines personnes pour la guérison ellemême. Mais la croyance à des propriétés curatives de l'opium existe aussi parmi la population. Cette idée est chère à certains historiens qui tendent à nuancer les ravages causés par l'opium. Ils soulignent que le délabrement physique de certains fumeurs provient en fait d'une maladie grave dont, en fumant la drogue, ils espèrent se guérir ou du moins neutraliser les souffrances ${ }^{39}$. Xun Zhou expose à nouveau dans un récent article l'importance de la consommation à but thérapeutique à la fin des Qing ${ }^{40}$. Un autre historien, Lars P. Laamann dresse de façon convaincante une longue liste de maladies, qui inclut peste et malaria, auxquelles les Chinois pensent alors que l'opium peut porter remède ${ }^{41}$. Nous avons pu constater qu'effectivement, en 1932, lors d'une forte poussée de choléra à Canton, un nombre important de personnes tombent subitement malades précisément dans les fumeries. Ceci s'explique fort bien par le fait que, confrontés

un exposé plus conséquent, voir Zhu Qingbao, Jiang Qiuming, Zhang Shijie, Yapian yu jindai zhongguo, p. 159-163 et 173-183 et R. K. Newman, art. cit., p. 776-78.

${ }^{37}$ Yuehuabao 18/7/30.

${ }^{38}$ Commission d'enquête sur l'opium en Extrême-Orient, interview du 20 janvier 1930 : archives de la SDN, dossier S 196.

${ }^{39}$ R. K. Newman, art. cit., p. 776.

${ }^{40}$ Xun Zhou, art. cit., p. 29.

${ }^{41}$ Lars P. Laamann, art. cit., p. 3 et 9. 
aux premiers symptômes de malaise, les malades ont le réflexe d'aller fumer quelques pipes dans l'espoir de les dissiper ${ }^{42}$.

On doit se garder, lorsqu'on aborde la question du motif de la consommation d'opium, d'accorder un crédit total aux déclarations des intéressés. En effet, en dépouillant systématiquement le Yuehuabao, on s'aperçoit que, lorsqu'un individu est pris en train de fumer en situation irrégulière (dans une fumerie clandestine par exemple), il met très souvent sur pied, pour s'expliquer, une histoire de maladie qui est parfois abracadabrante. Ceci semble indiquer que la consommation d'opium justifiée par une maladie subit une réprobation sociale beaucoup moins forte que celle qui vise à un pur désir de divertissement ${ }^{43}$. Cette situation devait vraisemblablement inciter les fumeurs admis à la clinique à prétexter un maladie pour se justifier. Or, près de $74 \%$ des patients de l'institut de 1937 déclarent être venus à la drogue pour le plaisir (wannong 玩弄) ${ }^{44}$. La consommation de loisir était donc très prépondérante à Canton dans ces années-là. Il s'agit peut-être d'une spécificité cantonaise, suggérée d'autre part par certaines données de la fin du XIX ${ }^{\mathfrak{e}}$ siècle ${ }^{45}$. En tout cas, ces chiffres donnent à réfléchir sur l'opinion de certains historiens qui affirment que les prises d'opium sont liées principalement à une médication, sans même prendre en compte la question de la maladie-alibi susceptible de biaiser les réponses des fumeurs ${ }^{46}$.

Il est très probable qu'à Canton la majorité des fumeurs entrent dans la carrière pour le plaisir ou du moins poussés, petit à petit, par leur entou-

\footnotetext{
${ }^{42}$ Yuehuabao 17/6/32, 20/6/32.

${ }^{43}$ Yuehuabao 5/6/31, 26/10/31, 4/12/31, 31/12/31, 9/3/34.

${ }^{44}$ GJYN, partie statistique, p. 5 . Les $26 \%$ restant prétendent avoir commencé la consommation d'opium pour soulager des maux (les maux d'estomac, la toux et les maladies vénériennes sont les trois principaux).

${ }^{45}$ Wang Shuhuai 王樹槐, "Yapian duhai, guangxu ershisan nian wenjuan diaocha fenxi » 鴉片毒害, 光緒二十三年文卷 調查分析 (Les ravages de 1'opium : analyse d'un questionnaire de 1897), Bulletin of the Institute of Modern History, Academia Sinica (Taiwan), 1980, n 9 , p. 189.

${ }^{46}$ Wang Shuhuai, ibid.
} 


\section{Xavier Paulès}

rage dans le cadre des loisirs ${ }^{47}$. Nombre d'articles du Yuehuabao, qui décrivent avec horreur les mœurs des fumeries, commencent par un petite introduction dans laquelle l'auteur se justifie en expliquant qu'il n'est pas un fumeur lui même et qu'il n'est entré dans la fumerie dont il est question que sur les instances d'un ami. C'est le cas par exemple dans l'extrait suivant $^{48}$ :

Un de mes amis du nom de Deng est un opiomane notoire. Hier je l'ai croisé et il a insisté pour que je le suive dans une fumerie pour passer le temps. Il m'a été impossible de refuser et je l'ai donc accompagné. Une fois arrivés, Deng prit possession d'une couche et commença à fumer comme un enragé, en expirant une abondante fumée.

友人鄧君, 瘾君子也, 昨遇於途, 力邀余偕往虎窝消遺, 卻辭不獲, 始相 隨同往，抵步，鄧擈榻狂吸，呵氣如雲。

Ce petit couplet, dont la sincérité est parfois sujette à caution, a néanmoins le mérite de montrer comment des personnes peuvent se trouver amenées à consommer de l'opium dans le cadre de leurs relations amicales. Phénomène largement attesté dans le Canton des années $1930^{49}$, la consommation d'opium dans des lieux de plaisirs qui, rappelons-le, ne sont pas uniquement des fumeries mais également des tripots ou surtout des lupanars, a des conséquences importantes sur le recrutement de nouveaux adeptes. Dans ces lieux se produisent maintes fois l'initiation de novices qui se trouvent entraînés par leurs amis.

${ }^{47}$ Zhujiang xingqi huabao 珠江星期畫報 (Hebdomadaire illustré de la Rivière des perles), $\mathrm{n}^{\circ} 20$ (n.d., ca 1928), p. 19, Xianggang gongshang ribao du 20/6/35.

${ }^{48}$ Yuehuabao 7/1/32. On trouve aussi des articles de ce genre dans les éditions du Yuehuabao du 11/2/32, 2/2/32.

${ }^{49} J u d u$ yuekan, $\mathrm{n}^{\circ} 91$ (août 1935), p. 19-21; Cun Shi 存實 (pseudonyme), “Yanhua xueleihua Chentang » 煙花血淚話陳塘 (Paroles et larmes de sang des fumeurs du quartier de Chentang), in Guangdong fengqing lu廣東風情錄 (Recueil du Guangdong galant), Hong Kong : Zhongyuan chubanshe, 1987, p. 268-270. Il est à noter aussi que dans les années 1930-1936 existe à Canton une sorte de permis provisoire d'une durée de 24 heures pour fumer l'opium dans les hôtels, les restaurants, ou les lupanars. 


\section{Un grand déséquilibre hommes / femmes}

La part des femmes dans la population des fumeurs d'opium ne peut être étudiée qu'en ayant conscience de la réprobation particulière dont font l'objet les fumeuses. Un article de Keith Mac Mahon montre, à partir d'un corpus d'œuvres littéraires de la fin des Qing, comment la fumeuse, comme virilisée par la drogue, paraît mettre en danger un ordre social traditionnel ${ }^{50}$. En tant que telle, la réprobation qui la touche est plus forte que celle visant ses congénères masculins. Certains articles du Yuehuabao suggèrent que tel est toujours le cas dans les années 1930. Un nombre important de femmes arrêtées pour consommation clandestine d'opium manifestent la honte qu'elles ressentent lors de leur transfert au poste de police, dissimulant leur visage ${ }^{51}$. Les mentions d'hommes qui manifestent leur confusion de cette façon quand ils sont arrêtés sont beaucoup plus rares et seulement le fait de certaines professions comme les enseignants ${ }^{52}$. Le relevé des faits divers liés à l'opium dans le Yuehuabao de 1930 à 1936 montre également que les histoires impliquant des femmes semblent plus dignes d'être rapportées. Les titres des articles précisent toujours qu'il s'agit de femmes dans le cas où la police met la main sur des fumeurs clandestins de ce sexe. Le Yuehuabao annonce ainsi : Juqu lüzhong zhi nüdaoyou 拘去旅中之女道友 (Arrestation d'une fumeuse dans un hôtel), ou encore : Funü xiyan beiju zhi langbei 婦女吸煙被拘之狼狽 (Déconfiture d'une femme arrêtée en train de fumer l'opium) ${ }^{53}$.

En conséquence, il convient de considérer avec beaucoup de prudence les pourcentages de femmes parmi les patients de nos deux cliniques de désintoxication, tant il est probable que les femmes rechignent à un traitement public. On n'est donc pas surpris qu'effectivement, seulement

\footnotetext{
${ }^{50}$ Keith Mac Mahon, art. cit., p. 131-135.

${ }^{51}$ Yuehuabao 10/5/30, 18/10/30, 5/6/31.

${ }^{52}$ Yuehuabao 6/3/32.

${ }^{53}$ Yuehuabao des $10 / 5 / 30$ et $5 / 6 / 31$. Il en existe beaucoup d'autres, dans les numéros $\operatorname{des} 18 / 10 / 30,22 / 10 / 30,1 / 2 / 34,27 / 4 / 33,10 / 7 / 33,28 / 9 / 33,22 / 9 / 35$.
} 


\section{Xavier Paulès}

$0,97 \%$ et $2,9 \%$ des patients des deux cliniques de désintoxication soient des femmes ${ }^{54}$. La comparaison, à la même époque, avec les chiffres des hôpitaux de Hong Kong, où le traitement des fumeurs n'est pas obligatoire, est éclairante : cette absence de contrainte semble inciter les femmes à ne pas se faire soigner. Par exemple, l'hôpital Tung Wah n'accueille aucune femme en 1934-1935, parmi ses 842 malades-fumeurs ${ }^{55}$. Un rapport du Consul de France à Hong Kong, en 1929, évaluait pourtant à 1 ou $2 \%$ la proportion de femmes qui fument dans la colonie britannique ${ }^{56}$.

De même, les données individuelles pour 171 patients, fournies par la clinique de 1941, laissent paraître une éloquente surreprésentation des femmes parmi les personnes envoyées de force (par opposition aux patients volontaires) se faire soigner par les bureaux de police des quartiers : elles en représentent $13,3 \%{ }^{57}$.

Les pourcentages de femmes parmi les patients des cliniques ne semblent donc, à l'évidence, pas représenter le vrai poids des femmes parmi les fumeurs. Les arrestations à Canton pour des faits liés au jeu clandestin ou à l'opium concernent les femmes à hauteur de 3,5\% pour la période de janvier 1928 à juin $1929^{58}$. Les chiffres des arrestations pour délits liés à l'opium de $19322^{59}$ montrent que sur 56 personnes arrêtées, 5 sont des femmes, soit $8,9 \%$. Ce chiffre de $8,9 \%$, malgré la modicité de

${ }^{54}$ Respectivement $0,97 \%$ des patients de la clinique de 1937 et $2,9 \%$ de celle de 1941.

${ }^{55}$ Report of the government of Hong Kong for the calendar year 1935 on the traffic in prepared opium. Report of the government of Hong Kong for the calendar year 1934 on the traffic in prepared opium, Hong Kong : Noronha \& Cie.

${ }^{56}$ Archives du Ministère des Affaires étrangères (MAE), Série SDN, Sous-série Affaires communes, dossier n ${ }^{\circ} 1642$ : Rapport du 12 décembre 1929 du consul de France à Hong Kong au Ministre des Affaires étrangères sur la question de l'opium à Hong Kong.

${ }^{57} G J L$, p. 30-40.

58 Guangzhoushi zhengfu 廣州市政府, Guangzhoushi zhengfu tongii nianjian 廣州市政府統計年鑒 (Annuaire statistique du gouvernement de la municipalité de Canton), 1929, p. 86-89.

${ }^{59}$ Zhonghua minguo tongji tiyao, p. 423. 
l'échantillon, se rapproche assez remarquablement des données nationales des criminels arrêtés pour faits liés à l'opium en 1931 qui donnent 2380 femmes sur un total de $27435(8,7 \%)^{60}$. Même s'il faut noter que ces deux dernières données concernent aussi bien les activités de contrebande (transport et vente clandestine) que la consommation, il n'en demeure pas moins que les cas de consommation illicite représentent de toute façon la très grande majorité des interpellations ${ }^{61}$.

Compte tenu des remarques concernant les chiffres trop faibles des cliniques de désintoxication, ces $8,9 \%$ constituent, même s'il faut rester très prudent, une estimation plus plausible du poids réel des femmes parmi les fumeurs cantonais. Si on la retient, en intégrant aussi le fait que la population de Canton compte environ 140 hommes pour 100 femmes à cette époque ${ }^{62}$, on peut donc avancer que les femmes fument environ sept fois moins que les hommes. En effet, une fois prise en compte la surreprésentation des hommes de la population de Canton, on obtient un rapport de $8,9 \times 1,4=12,46$ femmes pour 91,1 hommes, soit 7,3 fois moins.

En dehors de la réprobation sociale qui atteint les fumeuses, déjà évoquée, on peut avancer d'autres causes pour expliquer leur faible part : d'une manière générale, les femmes fréquentent moins les lieux de plaisir où l'on apprend à fumer. D'autre part, les femmes sont également moins présentes dans les catégories socio-professionnelles les plus concernées par la consommation d'opium, que nous allons aborder maintenant.

\footnotetext{
${ }^{60} \mathrm{Ibid}, \mathrm{p} .165$.

${ }^{61}$ Cette affirmation se base sur le constat dressé par la police cantonaise en 1922 (et qui demeure probablement vrai en 1931-32) : $80 \%$ des délits liés à l'opium concernent la consommation illicite et seulement $20 \%$ la vente et le transport d'opium de contrebande : Minguo ribao 民國日報 14/2/22.

${ }^{62}$ Guangzhoushi zhengfu tongii nianjian, p. 53. Il est à noter que ce déséquilibre hommes / femmes des populations urbaines est une constante de l'époque, et s'établit en moyenne à 143 hommes pour 100 femmes dans les six plus grandes villes de Chine en 1928 ; cf. Benshu bianjibu 本書編輯部, Zhonguo jingji nianjian 中國經濟年鍳 (Annuaire économique de la Chine), Shanghai : Benshu bianjibu chuban, 1934, partie C, p. 32.
} 


\section{Xavier Paulès}

\section{Métiers}

Ici encore, les chiffres fournis par l'institut de 1937 constituent une source de grand intérêt ${ }^{63}$, grâce à l'ampleur de l'échantillon et parce qu'on peut les croiser, malgré quelques différences de découpages des catégories professionnelles, avec les données concernant la population adulte masculine du recensement de Canton de $1928^{64}$. On supposera, bien sûr, qu'entre 1928 et 1937 ne sont pas apparus de grands changements au sein de la population active de Canton. (Voir graphiques $\mathrm{C}$ et $\mathrm{D}$ ).

Malgré toutes les précautions qu'impose la relative imprécision des catégories, on voit se dégager certaines caractéristiques. C'est d'abord le groupe des coolie qui paraît payer le tribut le plus important par rapport à son poids démographique dans la population globale $(6,6 \%$ de la population et $16,3 \%$ des malades). Le cas des ouvriers est comparable même si la surreprésentation est beaucoup moins spectaculaire $(42,7 \%$ de la population et $46,5 \%$ des malades). Les travailleurs manuels représentent donc $62,8 \%$ des fumeurs pour seulement $49,3 \%$ de la population adulte masculine : ils forment les gros bataillons des fumeurs.

Les paysans, qui paraissent également surreprésentés $(0,7 \%$ de la population et $4,1 \%$ des malades), posent un problème délicat car il est très probable que l'institut recrute un peu hors de l'agglomération proprement dite, ce qui pourrait gonfler artificiellement les chiffres.

Le cas des commerçants est intéressant car le compilateur des données de l'institut a été poussé à établir une distinction entre shang 商 (les marchands) et fan 販 (les colporteurs ou vendeurs à l'étal), distinction qui n'existe pas pour les données du recensement de $19288^{65}$. Cela s'explique certainement par le fait que la consommation d'opium semble être tout particulièrement importante parmi les fan en question $(11,9 \%$ des effectifs de

${ }^{63}$ GJYN, partie statistique, p. 2.

${ }^{64}$ Ces résultats sont publiés dans le Guangzhoushi zhengfu tongji nianjian, 1929, p. 53.

${ }^{65}$ Afin de pouvoir comparer catégorie par catégorie les deux graphiques, j'ai fondu les chiffres des fan et des shang dans un même ensemble intitulé 'marchands'. 
l'institut). Un article du Guangzhou zazhi 廣州雜誌 s'attarde d'ailleurs sur la consommation d'opium parmi les vendeurs à l'étal dans un article qui leur est en partie consacré ${ }^{66}$. Les commerçants sont donc une catégorie visiblement traversée horizontalement, dans son rapport à la consommation d'opium, par un net clivage lié au niveau d'aisance.

Les chiffres plutôt flatteurs concernant les militaires et les policiers sont à considérer avec circonspection étant donné que ces personnes ont peut être davantage la possibilité de se soustraire au traitement. Ces résultats peuvent être en effet dus à la qualité du recrutement et de la formation des soldats et policiers cantonais ${ }^{67}$ aussi bien qu'à une certaine impunité. Le climat de reprise en main de l'administration cantonaise après le retour dans le giron de Nankin en juillet $1936^{68}$, moins favorable au népotisme, ainsi que l'absence de toute allusion dans les sources à une quelconque prolifération des fumeurs parmi la police et les militaires de Canton incitent à privilégier la première hypothèse ${ }^{69}$.

La clinique ne fournit pas de précisions pour les professeurs, les fonctionnaires, les professions libérales, englobés dans la rubrique « autres » qui ne représente que $0,9 \%$ du total des patients. Le recensement de 1928, plus précis, nous apprend que ces trois catégories occupent respectivement $0,8 \%, 0,8 \%$ et $1,3 \%$ des hommes adultes, ce qui suffit

${ }^{66}$ Guangzhou zazhi 廣州雜誌, $\mathrm{n}^{\circ} 30\left(1^{\mathrm{er}}\right.$ juillet 1934), p. 15.

${ }^{67}$ Deux articles consacrés à la police de Canton de la China Weekly Review du 29 novembre 1930 et du 29 décembre 1928 donnent une impression très favorable de la police de Canton, de même que les passages consacrés à ce sujet dans $\mathrm{Ng}$ Yong Sang, Canton, city of the rams, Canton : M. S. Cheung, 1936, p. 22-23. Des louanges semblables du Consul anglais de Canton apparaissent dans un résumé des rapports confidentiels envoyés par les consuls en Chine et compilé par l'ambassade de Grande Bretagne en Chine au premier trimestre 1924, publié dans Robert L. Jarman, China, political reports 1911-1960, 11 vol., Archives Editions Limited, 2001, vol. 3, p. 23.

${ }^{68}$ Revue nationale chinoise, octobre 1936, p. 59, et décembre 1936, p. 303.

${ }^{69}$ À certaines autres périodes de l'époque républicaine, les militaires stationnés à Canton sont décrits par des sources assez sûres comme très concernés par la consommation d'opium. Ce n'est plus le cas dans les années 1930. 


\section{Xavier Paulès}

donc à montrer la moindre emprise de la consommation d'opium sur ces élites.

Pour synthétiser, la grande tendance qui se dessine est donc la suivante : plus l'on se situe bas dans la hiérarchie sociale et plus la proportion des fumeurs est élevée. Elle ne semble pas résulter de l'incapacité des classes les moins favorisées de la population à échapper au traitement car elle est confirmée par d'autres sources de l'époque : dès 1925, un rapport du Foreign Office à la Société des Nations ${ }^{70}$ relève que « les fumeurs se recrutent principalement parmi les coolies de palanquins, les traîneurs de pousse pousse et autre gens de même classe "). Dix ans plus tard, au moment de la reprise en main de la province par les autorités de Nankin, un rapport daté du 15 septembre 1936 et adressé à Chiang Kai-shek ${ }^{71}$ précise qu'au Guangdong, les fumeurs sont spécialement nombreux dans les couches les plus basses de la société et que parmi celles-ci, la fréquentation des fumeries peut être considérée comme presque habituelle. De même, selon un article de la revue $J u d u$ yuekan de 1929, au Guangdong, les fumeurs d'opium sont surtout nombreux dans les rangs des travailleurs ${ }^{72}$.

Il faut souligner que cette part prépondérante des catégories les plus modestes de la population cantonaise apparaît, à la lumière d'une comparaison avec la situation de la fin de l'Empire, comme une nouveauté due probablement au recul de la pratique parmi les classes les plus favorisées. Les sources diplomatiques et missionnaires de la fin de l'Empire soulignaient en effet la pénétration de cette pratique dans les élites fonctionnaires de Canton ${ }^{73}$. Dans un article daté de 1912 intitulé « Voyage autour de

\footnotetext{
${ }^{70}$ Dossier FO 228/3369 : daté du 12 mars, ce rapport reprend en fait un texte des services secrets britanniques pour la période d'avril à septembre 1924 à Canton qui se trouve, lui, dans le dossier FO 228/3368.

${ }^{71}$ Archives nationales $\mathrm{n}^{\circ} 2$ (Nanjing) : dossier 41/519.

${ }^{72}$ Judu yuekan, $\mathrm{n}^{\circ} 36$ (décembre 1929), p. 47.

${ }^{73}$ MAE, Nouvelle série, Sous-série Chine, dossier 650, Rapport médical annuel du poste de Canton de 1909; The Foreign field (Mensuel de la Wesleyan Methodist Church), juillet 1911, p. 334 ; cet article est, en fait, consacré au district de Zhongshan 中山, encore appelé 香! à l'époque.
} 
l'horizon cantonais ", un missionnaire de Canton, M. Gervaix, pouvait même évoquer le fumeur d'opium de cette façon: "Mais, si le sort du mendiant émeut la compassion, combien plus je déplore le sort du fumeur d'opium ! Car, s'il n'a pas, comme le précédent, la malchance du dénuement, il subit, par contre, les inconvénients d'une aisance qui tourne à la ruine de son existence et de sa renommée ${ }^{74}$ ». Pour Gervaix, le fumeur typique de 1912 est donc un personnage issu des catégories les plus favorisées de la société.

Les succès de la politique impériale de lutte contre l'opium à partir de 1906, qui obligeait les élites à donner l'exemple et interdisait aux fonctionnaires de continuer à fumer ${ }^{75}$, ajoutés à une meilleure prise de conscience des périls de la consommation et de l'idée que l'opium est à l'origine du déclin du pays ${ }^{76}$, ont largement détourné de la drogue les élites cantonaises. On constate que tel est toujours le cas dans les années 1930 : selon un rapport du Consul de France de 1932, « les fonctionnaires civils et militaires ainsi que les étudiants [ne sont] adonnés à cette pratique qu'exceptionnellement ${ }^{77}$ ).

La vision des contemporains, pour qui le fumeur typique des années 1930 n'est plus le riche marchand ou le fonctionnaire du père Gervaix mais un coolie, correspond bien à la réalité des chiffres que nous avons évoqués. Ce fait est probablement lourd de conséquences pour l'image du fumeur d'opium dans la société, surtout dans la mesure où la propagande antiopium de l'époque, dans ses affiches, appuie très habilement là où ça fait

\footnotetext{
${ }^{74}$ Gervaix, «Voyage autour de l'horizon cantonais », Les Missions catholiques, 1912, p. 358 .

${ }^{75}$ Report of the International anti-opium commission, Shanghai : North China Daily News, 1909, p. 72-76.

${ }^{76}$ MAE, Nouvelle série, Sous-série Chine, dossier $n^{\circ} 592$ : rapport du 21 août 1912 de Picot à Poincaré, Ministre des Affaires étrangères.

${ }^{77} \mathrm{MAE}$, Nantes, Pékin, Série $\mathrm{A}$, dossier $\mathrm{n}^{\circ} 157$ : rapport du consul de France à Canton du 15 décembre 1932.
} 


\section{Xavier Paulès}

mal en montrant toujours des fumeurs maigres et en haillons ${ }^{78}$. L'image des consommateurs d'opium, désormais associés au bas peuple est donc dévalorisée. Ceci contribue certainement à nourrir le désintérêt déjà observé parmi les jeunes, car ce n'est plus «chic » de fumer. Dans ces conditions, l'absence remarquable de témoignage littéraire ou d'écrit autobiographique de fumeurs, phénomène nouveau que l'on peut constater sous la République, est assez logique : les lettrés et les intellectuels fument peu, et ceux qui le font n'en sont pas fiers.

\section{Étude du cas des tireurs de pousse-pousse}

\section{Importance de la consommation parmi les tireurs de pousse}

Nous venons de voir que la proportion de fumeurs d'opium des années 1930 est particulièrement élevée parmi les travailleurs manuels et plus particulièrement les coolies. Or, le tireur de pousse est par excellence, pour les Cantonais des années 1930, l'incarnation du travailleur de force. Ceci explique en partie que nous allons nous intéresser à son cas. Mais, en dehors du fait qu'il représente une part significative des fumeurs, il offre aussi l'avantage d'attirer dans la période l'attention des élites politiques et intellectuelles ainsi que de la presse cantonaise. Les conditions de vie des tireurs suscitent en effet des travaux universitaires et des enquêtes officielles, ce qui n'est pas le cas pour d'autres fumeurs tels les policiers, les militaires ou les marchands. Le cas des tireurs de pousse, grâce à cette relative abondance de sources, va aussi permettre de nous dégager quelque peu de la sécheresse des seules données statistiques. On est effectivement en mesure de décrire leurs pratiques de consommation au quotidien, $d$ 'identifier

\footnotetext{
78 Voir les affiches publiées dans le Jinyan banyuekan 禁煙牛月刊 (bimensuel de la prohibition de l'opium) $\mathrm{n}^{\circ} 1$, juin 1936, ou encore celles du Jinyan zhuankan 禁煙專刊 de juin 1937.
} 
le type d'opium qu'ils consomment, comment ils le fument, et de s'immiscer dans les fumeries populaires qu'ils fréquentent.

Le lien fort entre les tireurs de pousse et la consommation d'opium est suggéré par un guide touristique japonais de 1928. Cet ouvrage met en garde ses lecteurs contre les tireurs de Canton, selon lui en général remarquablement lents et inefficaces, car pour la plupart fumeurs d'opium ${ }^{79}$. De même, dans certaines nouvelles publiées dans les années 1930 par l'écrivain cantonais Ouyang Shan 歐陽山, les fumeries sordides décrites sont peuplées de tireurs de pousse ${ }^{80}$.

Une vaste enquête consacrée à ces gens et menée à bien par la municipalité de Canton confirme la réalité de la pénétration de l'opium parmi eux. Elle porte sur un ensemble très conséquent de 5253 individus alors que les effectifs des tireurs de pousse cantonais au milieu des années 1930 sont compris entre 5000 et $6000^{81}$. Dans cette enquête, $18,2 \%$ des tireurs interrogés déclarent fumer (shihao yapian 嗜好鴉片, ce qui veut dire qu'il s'agit d'une consommation régulière) ${ }^{82}$. L'une des raisons que l'on peut avancer pour justifier cette proportion particulièrement élevée par rapport au reste de la population est qu'ils utilisent la drogue comme un remontant pour les soutenir dans leur harassant travail. Dans un entretien de 1991, un ancien tireur de pousse cantonais déclare que l'opium, de même que le ta-

79 Murayama Shigeru, 村山しげる, Kanton Sadan 広東頊談, Osaka：Kibunkan, 1928 , p. 86-87. On trouve également des allusions à la prégnance de la consommation d'opium parmi les tireurs de pousse dans Canton Gazette du 28/1 1/33 et dans la revue Judu yuekan, $\mathrm{n}^{\circ} 90$ (juin 1935), p. 7.

${ }^{80}$ Ouyang Shan 歐陽山, “Pigun shijie 》痞棍世界 (Un monde de canailles), in Owyang Shan wenji 歐陽山交集, Guangzhou : Huacheng chubanshe, 1988, vol. 1, p. 362-377.

${ }^{81}$ Fung Chi Ming, History at the grassroots: rickshaw pullers in the Pearl River delta of South China, 1874-1992, thèse de doctorat, Université de Hong Kong, 1996, p. 175 176.

${ }^{82}$ Guangzhou nianjian bianzuan weiyuanhui 黄州年鉒編纂委員會, Guangzhou Nianjian 廣州年鑒, 1935, partie consacrée à la société, p. 53. 


\section{Xavier Paulès}

ancien tireur de pousse cantonais déclare que l'opium, de même que le tabac ou l'alcool, procure l'illusion de dissiper la fatigue ${ }^{83}$. L'opium a donc pour certains tireurs de pousse la fonction d'un indispensable stimulant. Une autre raison invoquée par beaucoup de sources est que les entrepreneurs qui leur louent les véhicules et fournissent aussi le logement incitent les tireurs à consommer dans des fumeries installées couramment dans les locaux mêmes où ils sont logés ${ }^{84}$. On consomme là une sorte d'opium trafiqué, à très bas prix ${ }^{85}$, le yantiao 煙條. Le yantiao est fabriqué en réutilisant des cendres d'opium ${ }^{86}$, et il est roulé en forme de tige (d'où son nom) ${ }^{87}$. C'est un produit parfaitement adapté à la demande des tireurs. Il peut être fumé avec une pipe plus courte et moins onéreuse que pour l'opium normal. Il présente aussi l'avantage d'être plus fort et beaucoup plus rapide à fumer car tout prêt à la consommation, si bien que deux à trois minutes suffisent à un tireur pour soulager son manque, alors qu'il lui en faudrait dix fois plus en fumant de l'opium préparé classique ${ }^{88}$.

Dans certains cas extrêmes, des loueurs de pousse, qui suscitent tout particulièrement l'indignation de la presse de l'époque ${ }^{89}$, obligent les tireurs qu'ils logent à consommer chaque jour une quantité fixée de drogue. Mais il existe aussi certaines fumeries qui louent des pousses à des tireurs à qui elles offrent l'hébergement gratuit à partir d'une certaine quantité de drogue consommée quotidiennement ${ }^{90}$. Cette pratique présente pour elles

${ }^{83}$ Fung Chi Ming, History at the grassroots, p. 180.

${ }^{84}$ Xianggang gongshang ribao 25/2/35.

${ }^{85}$ En plus de son très bas prix, le yantiao a l'avantage de pouvoir être débité en très petite quantité alors que l'opium préparé doit forcément l'être dans les pots d'une taille réglementée et scellés avec la marque du monopole : cf. Yuehuabao du 25/6/33.

${ }^{86}$ La consommation de l'opium fournit, par curage du fourneau de la pipe après usage, un résidu réutilisable appelé en anglais le dross, en chinois yanshi 煙屎, yapianshi 鴉片粱, ou encore yanhui 煙灰.

${ }^{87}$ Renjianshi 人間世, $\mathrm{n}^{\circ} 38$ (20 octobre 1935), p. 19-21.

${ }^{88}$ Yuehuabao 10/4/34, 25/6/33.

${ }^{89}$ Yuehuabao 4/2/34, 9/3/34.

${ }^{90}$ Xianggang gongshang ribao 25/2/35. 
le grand avantage de fidéliser une clientèle de base, étant entendu d'autre part qu'il n'est guère coûteux de laisser ces hommes dormir durant la nuit sur les couches de l'établissement qui, de toute façon, ferme ses portes.

Utilisation courante comme remontant, offre de yantiao à la fois puissant et bon marché, existence de stratégies d'incitation à la consommation échafaudées par les loueurs de pousses et certaines fumeries : nous pensons que pour toutes ces raisons, la part des véritables opiomanes parmi les tireurs de pousse fumeurs est effectivement assez forte. On comprend donc très bien que ce type de fumeurs ait accaparé l'attention de la presse et des élites, largement opposées à la consommation d'opium. Il paraît donc possible que les $18,2 \%$ évoqués plus haut représentent une sousestimation de la proportion de fumeurs. Pourtant, certaines enquêtes de l'époque incitent à la prudence en suggérant que la pratique de fumer l'opium est inégalement répartie parmi les tireurs. De fait, ils constituent un groupe social dont l'apparente homogénéité cache des disparités liées à des origines géographiques différentes, encore accentuées par des différences de dialectes et de localisation de l'habitat. Ainsi, selon une enquête centrée sur les tireurs originaires de la région de Huizhou 惠州, logés dans le quartier de Dongdi 東堤, la consommation d'opium est surtout importante chez les tireurs originaires de Canton même ${ }^{91}$. D'autre part, cette même enquête nous permet de mieux mesurer l'impact de l'opium sur cette population et de mettre en évidence la plus grande importance de l'alcool en tant que stimulant. L'enquête de la municipalité de Canton, déjà évoquée, confirme que, plus généralement, parmi l'ensemble des tireurs cantonais, plus de $53 \%$ boivent (shihao jiu 嗜好酒) ${ }^{92}$.

\footnotetext{
${ }^{91}$ Xianggang gongshang ribao 16/3/36.

${ }^{92}$ Guangzhou nianjian, 1935, partie consacrée à la société, p. 53.
} 


\section{Xavier Paulès}

\section{Aperçu sur les fumeries populaires}

À Canton, en dehors des établissements qui leur sont spécifiques, les tireurs de pousse sont les clients assidus des fumeries les plus modestes : Dans certaines, on consomme le yantiao; ce sont, dans l'argot cantonais propre aux fumeurs, "les établissements de la chose dure ${ }^{93}$. Dans d'autres, c'est un opium préparé normalement qui est servi. Nous allons, au travers de quelques extraits significatifs de journaux cantonais, essayer de donner les éléments concrets qui caractérisent la vie quotidienne de ce genre de fumeries.

Même si les faits divers des journaux ont tendance à donner une image trop inquiétante des fumeries populaires, puisqu'ils sont forcément consacrés aux incidents qui y surviennent, il est certain que ce sont néanmoins des lieux propices à des échauffourées :

Le 8 à 14 heures, un fumeur nommé Liu Zhen se rend dans une fumerie située au 1 , rue Baoshun. Une fois fini tout son pot d'opium, il s'endort sur place. Le patron, Liu Huan, considérant qu'il gêne ses affaires, prétend le chasser. Mais Liu Zhen n'y consent pas, répond avec arrogance et une dispute entre les deux hommes s'ensuit, qui dégénère en bagarre. Liu Huan empoigne alors une pipe pour frapper Liu Zhen en pleine tête ${ }^{94}$.

八曰下午二時許, 煙人劉珍到寶順街一號談話室吸煙, 全盅吸完, 酣眠不

去，館主劉煥，以其有礙營業，將之斥逐，劉珍不服，反唇相譏，二人因 此發生口角, 繼而動武, 劉煥即順手執起煙槍, 向劉珍迎頭擊下。

Cet extrait illustre un classique des faits divers cantonais des années 1930 : les bagarres dans une fumerie à coup de pipes à opium, souvent la conséquence d'une dispute pour une place (les fumeurs en manque - ce n'est pas le cas de ce Liu Zhen - étant de surcroît particulièrement irrita-

\footnotetext{
${ }^{93}$ La «chose dure " est un autre nom pour désigner le yantiao, probablement parce qu'il ne se présente pas sous forme liquide comme c'est le cas pour la « chose molle», l'opium normal, préparé sans adjonction de cendres: Xianggang gongshang ribao $18 / 6 / 35$.

${ }^{94}$ Yuehuabao 10/5/30.
} 
bles). Celles-ci se produisent généralement lorsque l'affluence est la plus grande, c'est-à-dire à l'heure de la sieste et après le dîner. Dans notre exemple, la dispute porte sur la possibilité pour un fumeur de s'assoupir sur place une fois qu'il a fini de consommer (et donc de continuer à occuper une place). Si une tolérance existe en dehors des heures de pointe, ce droit est cependant remis en cause dès lors que de nouveaux fumeurs attendent leur tour.

Les lignes suivantes, extraites du quotidien Yuehuabao décrivent la façon dont un client est accueilli à son arrivée dans une fumerie :

Le 18, Tang Chang, 42 ans, originaire de Sanshui, sans emploi ni adresse connue, va fumer à la fumerie Sanleju située dans le quartier de Henan au 2, rue $\mathrm{Bu}$, à l'étage. Le serveur, un certain Zhou Xiang, reconnaissant en Tang un habitué, l'accueille avec prévenance, lui donne une pipe, un pot d'opium, et encaisse pour un montant de 4 jiao ${ }^{95}$.

三水人唐昌, 四十二歲無職業住址, 十八日到河南布街二號樓上三樂居吸 煙, 琴僮周祥以唐係熟客, 殷勤招待, 給以煙槍一支煙亮一盅, 已收欵四 角。

On peut constater qu'à son entrée le client reçoit d'un employé de la fumerie une partie seulement du matériel nécessaire à la consommation de la drogue (une pipe dont il devient responsable et une dose d'opium correspondant à la somme versée). En dehors de la pipe, le reste du matériel (lampe, plateau, oreiller de porcelaine, aiguille et curette), moins susceptible d'être volé, est en effet déjà disposé sur les couches. La pratique qui consiste à encaisser l'argent à l'avance est une règle assez générale qui souffre néanmoins quelques exceptions notamment lorsque le client dispose d'une ardoise dans sa fumerie habituelle.

Dans l'environnement familier de la fumerie où ils ont leurs habitudes, les clients apprécient aussi une ambiance faite de convivialité entre les fumeurs. Ceux-ci, une fois leur éventuel manque satisfait, se plaisent à rester y bavarder. On peut le voir dans ce récit d'un lecteur du Yuehuabao très

${ }^{95}$ Yuehuabao 21/11/31. 


\section{Xavier Paulès}

hostile à l'opium qui décrit une fumerie où il s'est trouvé néanmoins contraint d'entrer :

Les fumeurs sont couchés dans tous les sens, mélangés, à aspirer bruyamment. Ceux qui sont déjà sous l'effet de l'opium s'asseyent le long des lits, s'entretiennent de choses étranges et débitent des discours incohérents. [On a] la gorge irritée, les oreilles assommées de bruit, [et pour compagnie] des gens qui se donnent en spectacle. Vraiment, voilà un remarquable tableau des bizarreries des fumeurs d'opium ${ }^{96}$.

諸道友縱橫錯雜, 居居競響, 其電力運行者, 則箕踞床沿, 互談怪論, 大 放㷧詞, 煙㬋刺耳, 醜態百出, 誠一幅絕妙煙人百怪圖也。

Le bruit résultant de l'aspiration de la fumée chargée d'opium ainsi que l'opacité de l'atmosphère sont fréquemment dénoncés comme les signes de l'ambiance infernale des fumeries populaires. Quant aux sornettes débitées par les fumeurs, elles désignent souvent, dans la bouche des noninitiés, des conversations « techniques » à propos de l'opium consommé ou du matériel utilisé. Le témoignage ci-dessus illustre en tout cas, indépendamment du jugement de valeur qu'il comporte, l'affluence et la popularité de cette fumerie ainsi que la convivialité régnant entre les fumeurs, venus probablement aussi pour bavarder entre amis. Nul doute que ceux qui s'y attardent ne considèrent pas l'endroit comme irrespirable et trop bruyant mais comme un lieu agréable où passer des moments de loisir.

Au terme de cette brève description d'un groupe particulier de fumeurs que sont les tireurs de pousse ainsi que des fumeries les plus modestes dont ils constituent une partie de la clientèle, il convient de souligner le risque d'en retirer une image trop schématique des fumeurs d'opium cantonais. Il existe, en effet, des pratiques de consommation différentes chez les fumeurs plus aisés, qui fréquentent volontiers des établissements agréablement aménagés sur plusieurs étages, dotés d'un beau mobilier, de commodités telles que des ventilateurs électriques en été et du chauffage durant

\footnotetext{
${ }^{96}$ Yuehuabao 7/1/32.
} 
les jours froids d'hiver ${ }^{97}$. Le service y est parfois assuré par des jeunes filles qui proposent leur compagnie aux clients, apprêtent pour eux un opium de très bonne qualité et leur vendent fruits et cigarettes ${ }^{98}$. Ceux-là viennent à la fumerie souvent plus pour se rencontrer et passer quelques instants de détente que pour faire taire à la va-vite leur état de manque. Il faudrait aussi évoquer la consommation à domicile, ou encore dans des lieux publics qui ne sont pas des fumeries (tripots, lupanars, restaurants), sans oublier le cas important des fumeries clandestines.

Les pratiques de consommation sont donc diverses malgré le poids important des fumeries de bas étage qui répondent aux besoins des gros bataillons des fumeurs.

\section{Conclusion}

Nous pensons avoir montré une image assez nette, quoique succincte, de la population des fumeurs de Canton des années 1930. Sa caractéristique la plus évidente est une concentration des usagers chez les travailleurs manuels (coolies, ouvriers, colporteurs) d'âge mûr et de sexe masculin. Il y aurait aussi beaucoup à dire sur d'autres sous-groupes, en particulier les fumeurs aisés, mais une certaine synthèse est nécessaire.

Une autre limite de cette étude est que, se concentrant sur la période où les sources sont les plus riches, elle ne constitue, à quelques rares exceptions près, qu'un instantané. Une étude de l'évolution à plus long terme pourrait permettre de déterminer si la situation des années 1930 est bien le résultat d'un processus continu de paupérisation par désaffection des classes privilégiées, doublé d'un vieillissement ${ }^{99}$.

\footnotetext{
${ }^{97}$ Renjianshi, ${ }^{\circ} 38$ (20 octobre 1935), p. 19-21.

${ }^{98}$ Minguo ribao $27 / 7 / 31$.

${ }^{99}$ Il existe un certain nombre d'indices d'un significatif vieillissement des fumeurs par rapport à l'époque impériale. Pour n'en citer qu'un, l'âge moyen des patients d'un institut de désintoxication ouvert en 1881 par un missionnaire n'est que de 35,3 ans,
} 


\section{Xavier Paulès}

Si on considère le seul aspect socio-professionnel des fumeurs, les différences entre la fin de l'Empire et les années 1930 semblent en tout cas bien montrer que la consommation d'opium, revenue en force après la chute de l'Empire, n'a plus les mêmes caractéristiques qu'à la fin du XIX siècle. Sur ce plan, la période républicaine ne se caractérise donc pas par un simple retour à la situation d'avant les édits de 1906, « rechute " provisoire due aux troubles politiques. Au contraire, elle est riche d'évolutions dont les transformations de la population des fumeurs d'opium ne constituent du reste qu'une des facettes ${ }^{100}$.

Il est intéressant de rapprocher cette mutation de la population des fumeurs des succès de la politique anti-opium des communistes après 1949. Ces brillants résultats sont toujours expliqués à la seule lumière de facteurs politiques: unification du territoire national, disparition des concessions étrangères, action efficace du PCC, et soutien actif des masses ${ }^{101}$. Nous pensons néanmoins qu'on comprend mieux le succès de cette campagne anti-opium et en particulier sa rapidité lorsqu'on sait que le groupe social des fumeurs d'opium, vieillissant et surtout recrutant essentiellement dans les strates les plus pauvres de la population, a déjà acquis alors une image peu reluisante. Il se trouve donc d'autant moins apte à résister à l'action vigoureuse des communistes au début des années cinquante.

Il y a là, de toute évidence, une piste prometteuse à explorer. Cela requiert toutefois une analyse plus sérieuse sur la période de la guerre et de

contre 38,6 ans pour la clinique ouverte en 1941 : Imperial Maritime Customs, Special series $\mathrm{n}^{\circ} 4$, Opium, Shanghai, $1881 ; G J L$, p. 27-40.

${ }^{100}$ Parmi d'autres phénomènes nouveaux, on peut aussi citer la percée remarquable des drogues de synthèse comme la morphine ou l'héroïne : cf. Frank Dikötter, "A cultural history of the syringe in modern China", Twentieth-Century China, vol. 28, $\mathrm{n}^{\circ} 1$, Novembre 2002, p. 37-56.

${ }^{101}$ Ezra Vogel, Canton under Communism programs and politics in a provincial capital, 1949-1968, New York : Harper \& Row, 1969, p. 31, 65-67, 88 ; Wang Hongbin, op. cit., p. 478-490; Su Zhiliang, op. cit., p. 466-471. 
l'occupation japonaise, uniformément traitée par les historiens chinois, pour la question de l'opium comme pour tant d'autres, par la compilation des atrocités, abus et exactions en tous genres des Japonais et de leurs 《 chiens courants » zougou 走狗 ${ }^{102}$.

${ }^{102}$ Un exemple de ce type de production : Li Enhan 李恩涵, «Riben zai huanan de fandu huodong 1937-1945 》日本在華南的販瑇活動, Bulletin of the Institute of Modern History, Academia Sinica (Taiwan), $\mathrm{n}^{\circ} 31$ (1989), p. 135-165. Dans sa communication au colloque Reinterpreting the Chinese city in the twentieth century à Lyon (1214 décembre 2002), Virgil Ho a montré tout l'intérêt et la nécessité d'un traitement plus nuancé de l'histoire du Canton de l'époque de l'occupation japonaise. 
Graphique A : Comparaison de la structure par âge de la population des patients de l'institut de désintoxication de 1937 avec celle de la population cantonaise masculine de 1928.

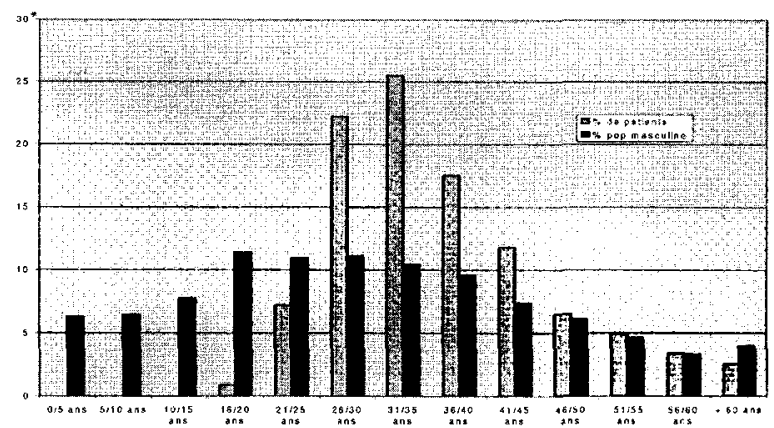

Sources : Guangzhoushi jinyan weiyuanhui, Guangzhoushi jinyan weiyuanhui gongzuo jiyao, Canton, 1937, et Guangzhoushi zhengfu tongji nianjian, 1929.

Graphique B : Comparaison de la structure par âge de la population des patients de l'institut de désintoxication de 1941 avec celle de la population cantonaise masculine de 1928.

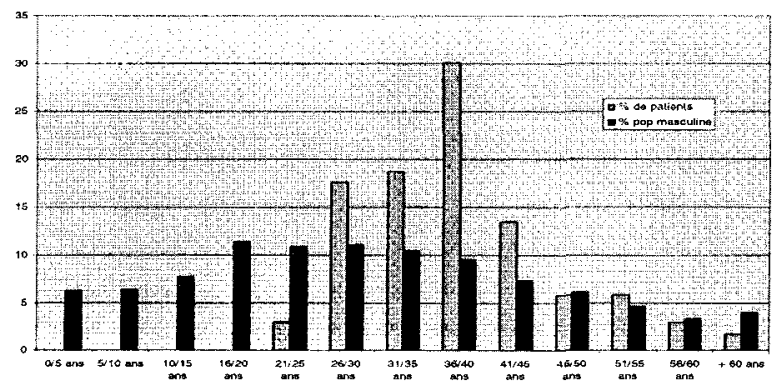

Sources: Guangdong jinyanlizyisuo chengli yi zhou nian jinian tekan, 1942, et Guangzhoushi zhengfu tongii nianjian, 1929. 
Graphique C : répartition par profession de la population des patients de l'institut de désintoxication de 1937.

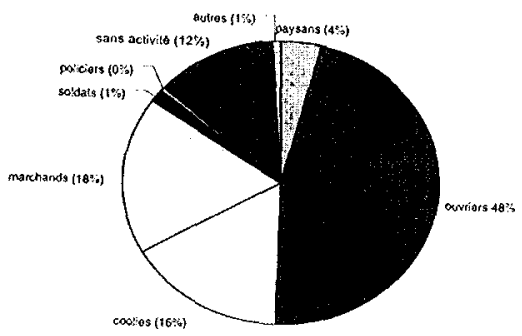

Source : Guangzhoushi jinyan weiyuanhui, Guangzhoushi jinyan weiyuanhui gongzuo jiyao, Canton, 1937.

[NB : colporteurs et marchands ont été regroupés sous la rubrique «marchands » afin de mieux comparer avec le recensement de 1928 qui ne fait pas cette distinction].

Graphique D : répartition par catégories professionnelles de la population cantonaise masculine adulte en 1928.

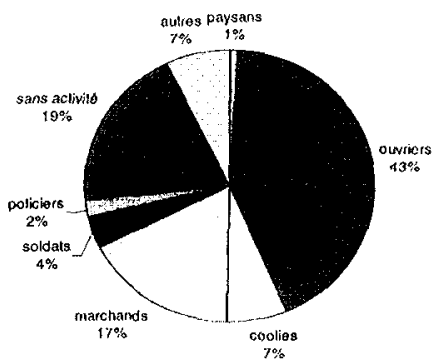

Source : Guangzhoushi zhengfu tongii nianjian, 1929. 


\section{Bibliographie}

Alan Baumler, Playing with fire: the nationalist government and opium in China, 1927-1941, thèse de doctorat, Université de l'Illinois, 1997.

Timothy Brook, Bob Tadashi Wakabayashi (éd.), Opium regimes, China, Britain and Japan, 1839-1952, Berkeley: University of California press, 2000.

Cun Shi 存實 (pseudonyme), «Yanhua xueleihua Chentang 煙花血淚話 陳塘 " (Paroles et larmes de sang des yanhua du quartier de Chentang), in Guangdong fengqing $l u$ 櫎東風情錄 (Recueil du Guangdong galant), Hong Kong : Zhongyuan chubanshe, 1987.

Frank Dikötter, "A cultural history of the syringe in modern China", Twentieth-Century China, vol. 28, n 1, Novembre 2002 p. 37-56.

Fung Chi Ming, History at the grassroots: rickshaw pullers in the Pearl River delta of South China, 1874-1992, thèse de doctorat, Université de Hong Kong, 1996.

Robert L. Jarman, China, Political Reports 1911-1960, 11 volumes, Archives Editions Limited, 2001.

Lars P. Laamann, "A history of narcotic consumption in modern China", Twentieth-Century China, vol. 28, $\mathrm{n}^{\circ} 1$ (novembre 2002) p. 1-20.

Li Enhan 李恩涵, "Riben zai huanan de fandu huodong 1937-1945 日本在華南的販毒活動》 (Les activités de trafic d'opium du Japon en Chine du sud entre 1937et 1945), Bulletin of the Institute of Modern History, Academia Sinica (Taiwan), 1989, n 31, p. 135-165.

Keith Mac Mahon, "Opium and sexuality in late Qing fiction", Nannü (2.1), 2000, p. 129-179.

R. K. Newman, "Opium smoking in late imperial China: A reconsideration", Modern Asian Studies, vol. 29 (4) (octobre 1995), p. 765-794.

Ouyang Shan 歐陽山, 《Pigun shijie 痁棍世界》(Un monde de canailles), in Ouyang Shan wenji 歐陽山交集 (Euvres de Ouyang Shan), Guangzhou : Huacheng chubanshe, 1988, vol. $n^{\circ} 1$, p. 362-377. 
Xavier Paulès, " Guérir, éduquer, délimiter : la clinique pour opiomanes de Canton en $1937 »$, Les cahiers d'histoire, 2002, n 3-4.

Edward R. Slack, Opium, State and society, China's narco-economy and the Guomindang, 1924-1937, Honolulu : University of Hawaï press, 2001.

Su Zhiliang 蘇智良, Zhongguo dupin shi 中國毒品史 (Histoire des stupéfiants en Chine), Shanghai : Renmin chubanshe, 1997.

Ezra Vogel, Canton under Communism programs and politics in a provincial Capital, 1949-1968, New York : Harper \& Row, 1969.

Wang Hongbin 王宏斌, Jindu shinian 禁毒史鍳 (Histoire de la prohibition des drogues), Changsha : Yuelu sushe, 1997.

Wang Shuhuai 王樹槐, "Yapian duhai, guangxu ershisan nian wenjuan diaocha fenxi » 鴉片毒害光緒二十三年交卷調查分析 (Les ravages de l'opium : analyse d'un questionnaire de 1897), Bulletin of the Institute of Modern History, Academia Sinica (Taiwan), 1980, ${ }^{\circ} 9$, p. $183-200$.

Xun Zhou, "Pain and pleasure: opium as medicine in late imperial China", Twentieth-Century China, vol. 28, $\mathrm{n}^{\circ} 1$ (novembre 2002), p. 21-36.

Yongming Zhou, Anti-drug crusades in twentieth-century China: nationalism, history and state building, Lanham : Rowman \& Littlefield, 1999.

Zhu Qingbao 朱慶葆, Jiang Qiuming 蔣秋明, Zhang Shijie 張士杰, Yapian yu jindai Zhongguo 鴉片與近代中國 (L'opium et la Chine contemporaine), Nanjing : Jiangsu jiaoyu chubanshe, 1995.

\section{Sources}

Archives de la SDN, dossiers S 196 et S 197.

Archives du Foreign Office, dossier FO 228/3369.

Archives du Ministère des Affaires étrangères (MAE), Nouvelle Série, Sous série Chine, dossiers $n^{\circ} 592$ et 650 .

Archives du Ministère des Affaires étrangères (MAE), Nantes, Pékin, Série A, dossier $n^{\circ} 157$. 


\section{Xavier Paulès}

Archives nationales $\mathrm{n}^{\circ} 2$ (Nanjing), dossiers 41/78, 41/519.

Benshu bianjibu 本書編輯部, Zhongguo jingji nianjian 中國經濟年鑒 (Annuaire économique de la Chine), Shanghai : Benshu bianjibu chuban, 1934.

Canton Gazette, Canton.

China Weekly Review, Shanghai.

Guangdong jinyan liuyisuo 廣東禁煙留醫所, Guangdong jinyan liuyisuo chengli yi zhounian jinian tekan, 廣東禁煙留醫所成立一週年紀念 特刊 (Édition spéciale pour commémorer le premier anniversaire de la fondation de l'institut de désintoxication du Guangdong), Canton, 1942 (avril).

Guangzhou nianjian bianzuan weiyuanhui 廣州年鍳編纂委員會, Guangzhou Nianjian 廣州年鍳 (Annuaire statistique de Canton), Canton, 1935.

Guangzhoushi jinyan weiyuanhui 廣州市禁煙委員會, Guangzhoushi jieyan yiyuan nianbao 廣州市戒煙醫院年報 (Rapport annuel sur la clinique de désintoxication de Canton), Canton, 1937.

Guangzhoushi jinyan weiyuanhui 廣州市禁煙委員會, Guangzhoushi jinyan weiyuanhui gongzuo jiyao 廣州市禁煙委員會工作紀要 (Résumé des activités du comité de suppression de l'opium de la ville de Canton), Canton, 1937.

Guangzhoushi zhengfu 廣州市政府, Guangzhoushi zhengfu tongji nianjian 廣州市政府統計年鑒 (Annuaire statistique du gouvernement de la municipalité de Canton), Canton, 1929.

Guangzhou zazhi 廣州雜誌 (La revue de Canton), Canton.

Jinyan banyuekan 禁煙牛月刊 (Bimensuel de la prohibition de l'opium),

Shanghai.

Jinyan zhuankan 禁煙專刊 (Édition spéciale pour la prohibition de l'opium), Shanghai.

Judu yuekan 拒毒月刊 (Mensuel pour la suppression des drogues), Shanghai. 
Murayama Shigeru 村山しげる, Kanton Sadan 広東瑻談 (Guide de Canton), Osaka : Kibunkan, 1928.

Les Missions catholiques, Paris.

$\mathrm{Ng}$ Yong Sang, Canton, city of the rams, Canton : M. S. Cheung, 1936.

Renjianshi 人間世 (Le monde terrestre), Hankou.

Report of the government of Hong Kong for the calendar year - on the traffic in prepared opium, Hong Kong : Noronha \& Cie.

Report of the International anti-opium commission, Shanghai : North China Daily News, 1909.

Revue nationale chinoise, Pékin.

The Foreign Field, Londres.

Xianggang gongshang ribao 香港工商日報 (Quotidien de l'industrie et du commerce de Hong Kong), Hong Kong.

Xunhuan ribao 循環日報 (La circulation), Hong Kong.

Yuehuabao 越華報 (Le méridional), Canton.

Zhonghua guomin juduhui 中華國民拒毒會, Zhongguo Yanhuo Nianjian 中國煙禍年鑒 (Annuaire des ravages de l'opium), Shanghai, 1931.

Zhonghua minguo tongji tiyao 中華民國統計提要 (Résumé statistique de la république de Chine), Shanghai : Shangwu yinshuguan, 1935.

Zhujiang xingqi huabao 珠江星期畫報 (Hebdomadaire illustré de la Rivière des perles), Canton. 


\title{
Xavier Paulès
}

\begin{abstract}
Résumé
Xavier PAULÈS : Les fumeurs d'opium à Canton dans les années 1930

L'existence de sources assez abondantes dans le Canton des années 1930 rend possible une étude de la population des fumeurs d'opium. Si ses effectifs sont finalement assez limités, la consommation d'opium a néanmoins la particularité de se concentrer dans certaines franges de la population, essentiellement parmi des hommes, travailleurs manuels, d'un âge compris entre 25 et 45 ans. Les femmes, la jeunesse et les élites sont au contraire notoirement imperméables à cette pratique. Les tireurs de pousse-pousse représentent l'archétype d'un groupe social fortement touché par l'opium, et l'étude de leur cas montre assez clairement quelle place cette drogue occupe dans leur vie quotidienne, tant comme un stimulant utilisé pour combattre la fatigue que comme un élément aliénateur dans leurs rapports avec les entrepreneurs qui leur fournissent véhicules et logements. Contrairement à l'époque impériale, la consommation d'opium dans le Canton des années 1930 est donc fortement associée à la catégorie des coolies, aussi bien statistiquement que dans leur représentation sociale.
\end{abstract}

\begin{abstract}
Xavier PAULÈs : Opium smokers in Guangzhou in the 1930s

The abundance of sources concerning Guangzhou in the 1930 decade enable a detailed study of opium smokers population. The figures as regards their number is not so impressive ; they concentrated in certain specific strata of the population, especially male manual workers in the 25-45 age bracket. Women, young people and upper classes were not part of this phenomenon. Rickshaw pullers examplify best the epitome of the smoker at this period, and the study of this category of the population demonstrates the importance of opium consumption as an energizer to compensate physical exhaustion, but also as a major stake in the relationship between the pullers and their patrons providers of their rickshaws and housing. Unlike the late Qing period, opium consumption was clearly connected to the coolies, not only in the reality of the statistics, but also in the collective representations.
\end{abstract}

